\section{On Cellular Darwinism: Mitochondria}

\begin{abstract}
The significant role of mitochondria within cells is becoming increasingly clear. This letter uses the NKCS model of coupled fitness landscapes to explore aspects of organelle-nucleus coevolution. The phenomenon of mitochondrial diversity is allowed to emerge under a simple intracellular evolutionary process, including varying the relative rate of evolution by the organelle. It is shown how the conditions for the maintenance of more than one genetic variant of mitochondria are similar to those previously suggested as needed for the original symbiotic origins of the relationship using the NKCS model.
\end{abstract}

\section{Larry Bull*}

University of the West of England

\section{Keywords}

Cellular Darwinism, coevolution, cytoplasm, mitochondria, NKCS model, organelle

\title{
I Introduction
}

Darwinian evolution has been either demonstrated or suggested as occurring within organisms in a number of ways, including in the immune system (e.g., [5]), in brains (e.g., [6]), and during development (e.g., [11]). Issa [7] has recently suggested that genomic areas with hypervariable DNA methylation provide a mechanism through which evolution may occur within the nucleus, potentially explaining some age-related cancers, for example. This letter explores another possible form of such cellular Darwinism, within the cytoplasm of eukaryotic cells by mitochondria.

It is well established that mitochondria were originally free-living bacteria that became organelles through the process of symbiogenesis (e.g., [12]). As well as providing energy through ATP, they are involved in the regulation of a number of critical cell functions such as cell division and the production of steroids, ammonia, and so on. This is perhaps somewhat to be expected, as it has long been noted that symbioses typically exhibit a positive feedback loop wherein beneficial adaptations in any partner(s) further increases their combined rate of selection (e.g., [10]). Mitochondria are typically inherited from the female and maintained in varying numbers (from one up into the thousands) depending upon the cell type and environment. Moreover, even egg cells typically contain more than one genetic variant of mitochondrial DNA. That is, cells maintain a genetically diverse population of mitochondria whose number can be varied during the cell's lifetime through the regulation of their reproduction and/or deletion. Thus, in view of the unavoidable copying errors occurring during reproduction (fission), a Darwinian process can be suggested to exist in the cytoplasm. For an effective selection process to occur from the cell's perspective, while it does not necessarily need to know which mitochondria are the current "best," it must be able to determine approximately whether increasing the number (concentration) of a particular genetic variant is having beneficial or detrimental effects

\footnotetext{
* Department of Computer Science \& Creative Technologies, University of the West of England, Bristol BSI6 IQY, United Kingdom. E-mail: Larry.Bull@uwe.ac.uk
} 
upon its current well-being. No exact mechanism for this ability is known to the author; for example, while it is likely to be one or more processes handled by the cell nucleus or involved in the maintenance/formation of mitochondrial networks (through fission and fusion), aspects of it may involve persisting quorum-sensing-like capabilities in the mitochondria. Note also that we assume that the cell's ability to regulate organelle reproduction and deletion provides a mechanism able to (roughly) target specific variants either spatially and/or genetically — for example, to initiate deletion (or possibly fusion) of the mitochondrion it last signaled to reproduce if it appears to be having negative effects.

This letter explores the conditions under which multiple genetic variants are maintained under such a cellular Darwinian process. This is done using a version of Kauffman and Johnsen's [9] abstract NKCS model, which allows for the systematic alteration of various aspects of a coevolving environment.

\section{The NKCS Model}

Kauffman and Johnsen [9] introduced the abstract NKCS model to enable the study of various aspects of coevolution. In their model an individual is represented by a genome of $N$ (binary) genes, each of which depends epistatically upon $K$ other randomly chosen genes in its genome. Thus increasing $K$, with respect to $N$, increases the epistatic linkage, increasing the ruggedness of the fitness landscapes by increasing the number of fitness peaks, which increases the steepness of the sides of fitness peaks and decreases their typical heights. Each gene is also said to depend upon $C$ randomly chosen traits in each of the other $S$ species with which it interacts. The adaptive moves by one species may deform the fitness landscape(s) of its partner(s). Altering $C$, with respect to $N$, changes how dramatically adaptive moves by each species deform the landscape(s) of its partner(s). The model assumes all inter- and intragenome interactions are so complex that it is appropriate to assign random values to their effects on fitness. Therefore for each of the possible $K+(S \times C)$ interactions, a table of $2^{K+(S \times C)+1}$ fitnesses is created for each gene, with all entries in the range 0.0 to 1.0 , such that there is one fitness for each combination of traits. The fitness contribution of each gene is found from its table; these fitnesses are then summed and normalized by $N$ to give the selective fitness of the total genome for that species. Such tables are created for each species (Figure 1; the reader is referred to [8] for full details).

Kauffman and Johnsen's [9] model uses populations of one individual (said to represent a converged species) and a genetic hillclimber to evolve them in turn. That is, if a given single gene mutant is found to be fitter than its parent in the current context of the other species, that species as a

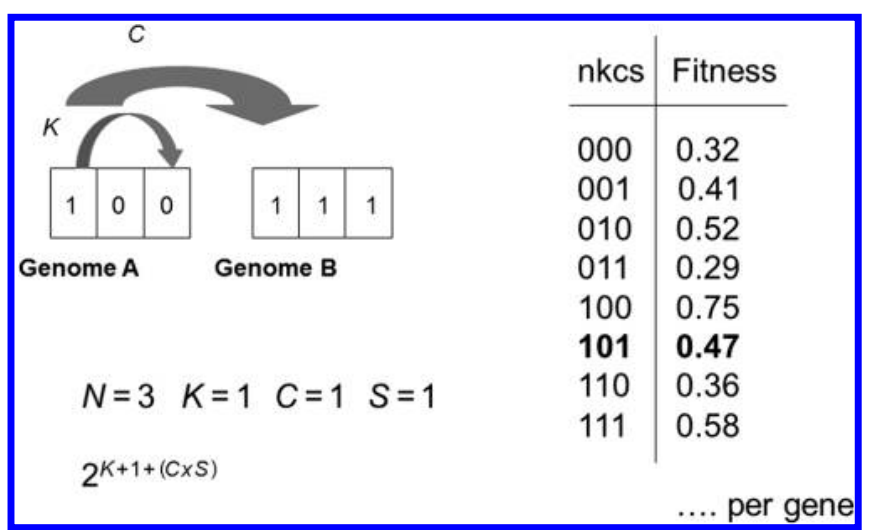

Figure I. The NKCS model: Each gene is connected to $K$ randomly chosen local genes and to $C$ randomly chosen genes in each of the $S$ other species. A random fitness is assigned to each possible set of combinations of genes. These are normalized by $N$ to give the fitness of the genome. 
whole moves to the configuration represented by the mutant. This is repeated for all species over a number of generations. They show how both inter- $(C)$ and intragenome $(K)$ epistasis affect a coevolving system, particularly in the attainment of Nash equilibria ("a combination of actions by a set of agents such that, for each agent, granted that the other agents do not alter their own actions, its action is optimal" [8, p. 245]). Their model is used here with three species, two of which represent the eukaryote with its mitochondria and the other the cell's environment (after [4]). A hillclimber is used with a probabilistic mutation rate of $1 / N$ per gene and $N=100$. All results presented are from 10 runs on each of 10 randomly created NKCS models, making 100 runs, after 100,000 generations. Gene values are initialized at random.

\section{An NKCS Model of Mitochondrial Intracellular Evolution}

Following [4], three species exist within the model — the nucleated genome, the mitochondria, and an external environment. For simplicity, each has the same internal epistasis $K$. The dependence between the genome and mitochondria is symmetrical and denoted by $C$, with a symmetrical degree of dependence between the genome and the external environment denoted by $E$ (Figure 2). Thus each gene in the genome has a fitness table of size $2^{K+C+E+1}$, in the mitochondria they are of size $2^{K+C+1}$, and in the environment species they are of size $2^{K+E+1}$. A cell is initiated with one mitochondrion.

The fitness of a cell is determined using an extension of the NKCS method described above, firstly for the nucleated genome with each mitochondrion present. This value is then averaged, that is, divided by the number of mitochondria it is partnered with. The fitness contribution of each mitochondrion is then calculated, and the result is also averaged. The fitness of the cell is the average of those two fitness contributions- the genome and mitochondria. An extra process to capture the lifetime evolution of the cytoplasm is then undertaken. A mitochondrion is picked at random from within the cell's population and (randomly) either copied with mutation or deleted (unless only one is present). The fitness of the cell with the changed number of mitochondria is then calculated. If the fitness is increased (decreased), the new mitochondria mix is adopted (not adopted). If the fitness is the same, then the configuration with the fewest mitochondria is kept-hence, there is a selective pressure against increasing their number - otherwise the decision is random. If the new configuration is adopted, the fitness of the cell is updated as the average of the original and the new fitness value; an assumption is made of equal lifetime in the original and the new state, whereas any nonadaptive states are quickly discarded and so are taken to have insignificant effect on lifetime fitness.

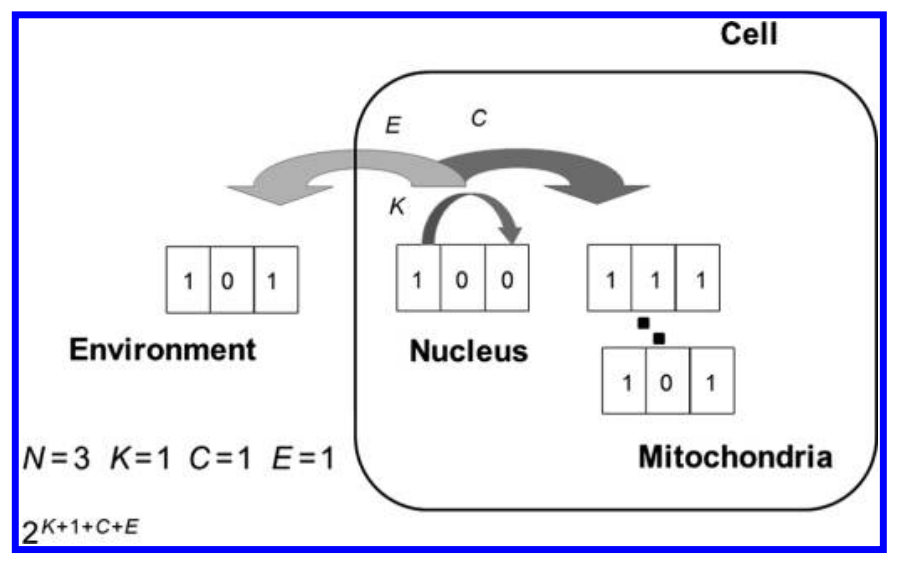

Figure 2. The NKCS model applied to represent a simple cell, with mitochondria, within an (evolving) external environment. For clarity, the fitness contribution calculation for only one gene in the nucleus is shown. 
A generation is said to have occurred when each part of the cell has been copied under mutation and evaluated as described, and the possible selective increase or decrease in mitochondria has been performed. The conditions for replacing the parent with the offspring are the same as those used to determine whether a new configuration of mitochondria is adopted. The environmental species is then evaluated with the current cell, produces an offspring under mutation, and adopts the offspring if its fitness is higher (ties broken randomly).

\section{Results}

Figure 3 shows how the average number of mitochondria seen in a cell (after 100,000 generations) rises above 1 with increasing frequency as $C$ increases, that is, with increasing dependence between the genome and mitochondria, when $E=1$. A previous study using the NKCS model of the conditions under which symbiogenesis may occur between a proto-eukaryote and free-living mitochondria found $C>K$ as the critical region [4]. Results here indicate that the average number of mitochondria rises from 1 roughly when $K+C \geq 5$. It can be noted that the number also rises for $C=1$ when $K>6$ (not shown). Therefore there is significant overlap, in the attribute space, between the region where the original symbionts merge and the region where mitochondrial diversity is maintained.

The cellular Darwinism mechanism, as proposed, represents a very simple form of cell lifetime learning. A study of the Baldwin effect [1] using the related NK model (single species; see [8]) found that the benefits of such learning can increase with increasing $K$ [2], that is, as the degree of ruggedness
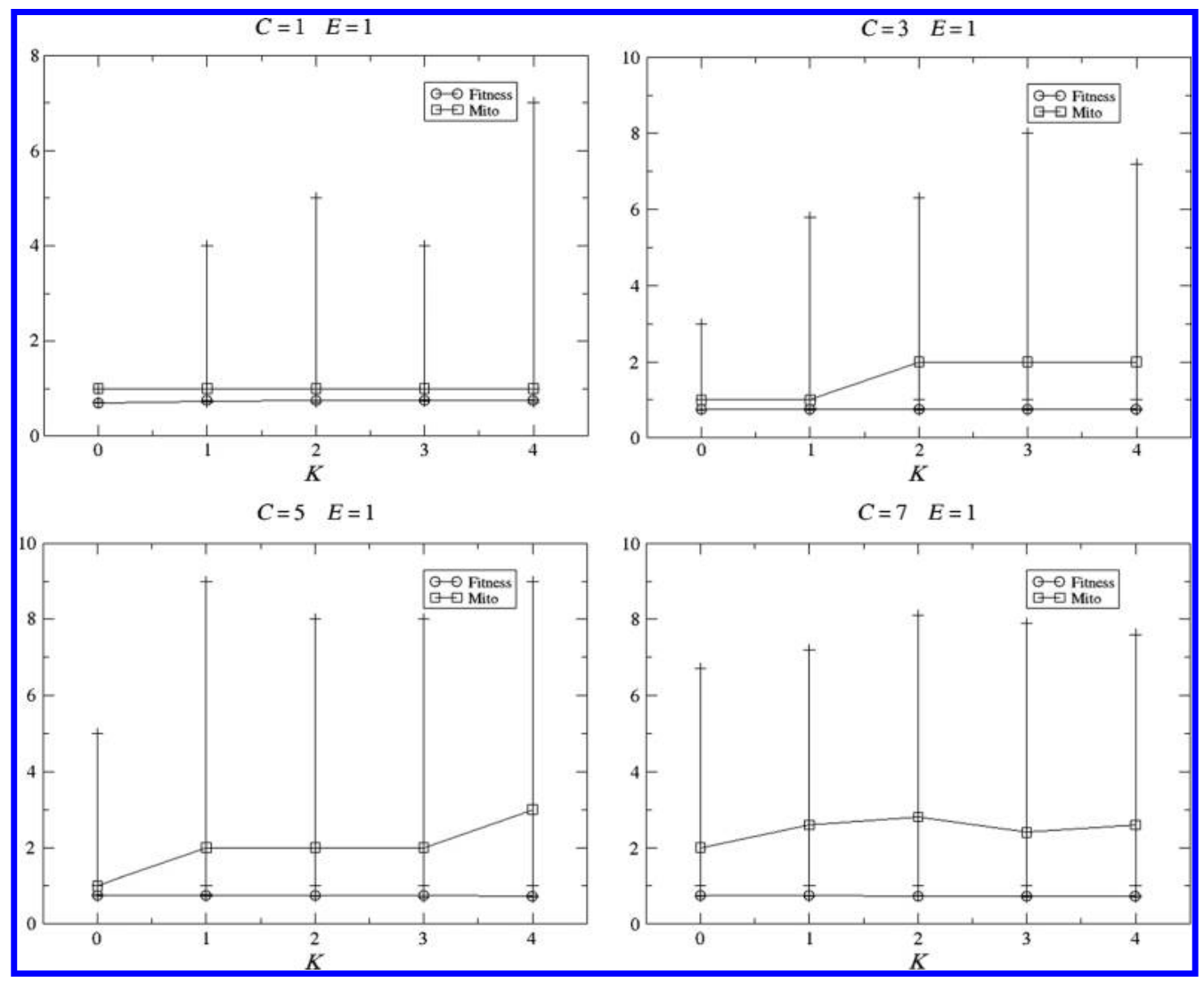

Figure 3. Example fitnesses and numbers of mitochondria in a cell after 100,000 generations across a variety of epistatic conditions within $(K)$ and between $(C)$ the nuclei/organelles, with low coupling to environment $(E=I)$. Error bars show min and max values. 
in the fitness landscape increases. That is, learning can aid evolution by effectively smoothing the underlying landscape. Since $K+C$ represents the internal epistasis of the cell [4], the finding here that it is adopted for higher combinations suggests that the mechanism can be seen to work in a similar way to the Baldwin effect; cell fitness becomes an average over a sample of genotypes in organelle space.

Figure 4 shows how the benefits of the learning process are not seen when the cell is more $(E=5)$ or less $(E=0)$ coupled to the coevolving environment. That is, the cellular Darwinism appears unable to cope with higher amounts of movement in the cell's fitness landscape, and is not sufficiently beneficial when there is no movement, respectively. Recall that there is a selective pressure against increasing the number of mitochondria.

In the above model, one generation of cytoplasm evolution is taken to occur during the lifetime of the cell. Following [3], where it was shown that increasing the rate of evolution in the free-living mitochondria relative to that in the proto-eukaryote expanded the conditions under which merging is likely to occur, the effects of increasing the number of generations to $M>1$ have been explored. Indeed, many cycles of mitochondria reproduction (and fusion) can occur within cells, particularly during the maintenance of the networks of the organelle (e.g., see [13]). Figure 5 shows that significant increases in the number of mitochondria ( $t$-test, $p<0.05)$ emerge for $E=0$ when $M=10$, compared to $M=1$ (Figure 4). Analysis of the resulting fitness levels shows a significant drop for $C=1$ and $K<2(t$-test, $p<0.05)$ and a significant increase for all $K$ when $C>3$, that is, for higher cellular epistasis (not shown). This also relates to the aforementioned study of the Baldwin effect, which showed how both the amount and frequency of learning affected performance [2].

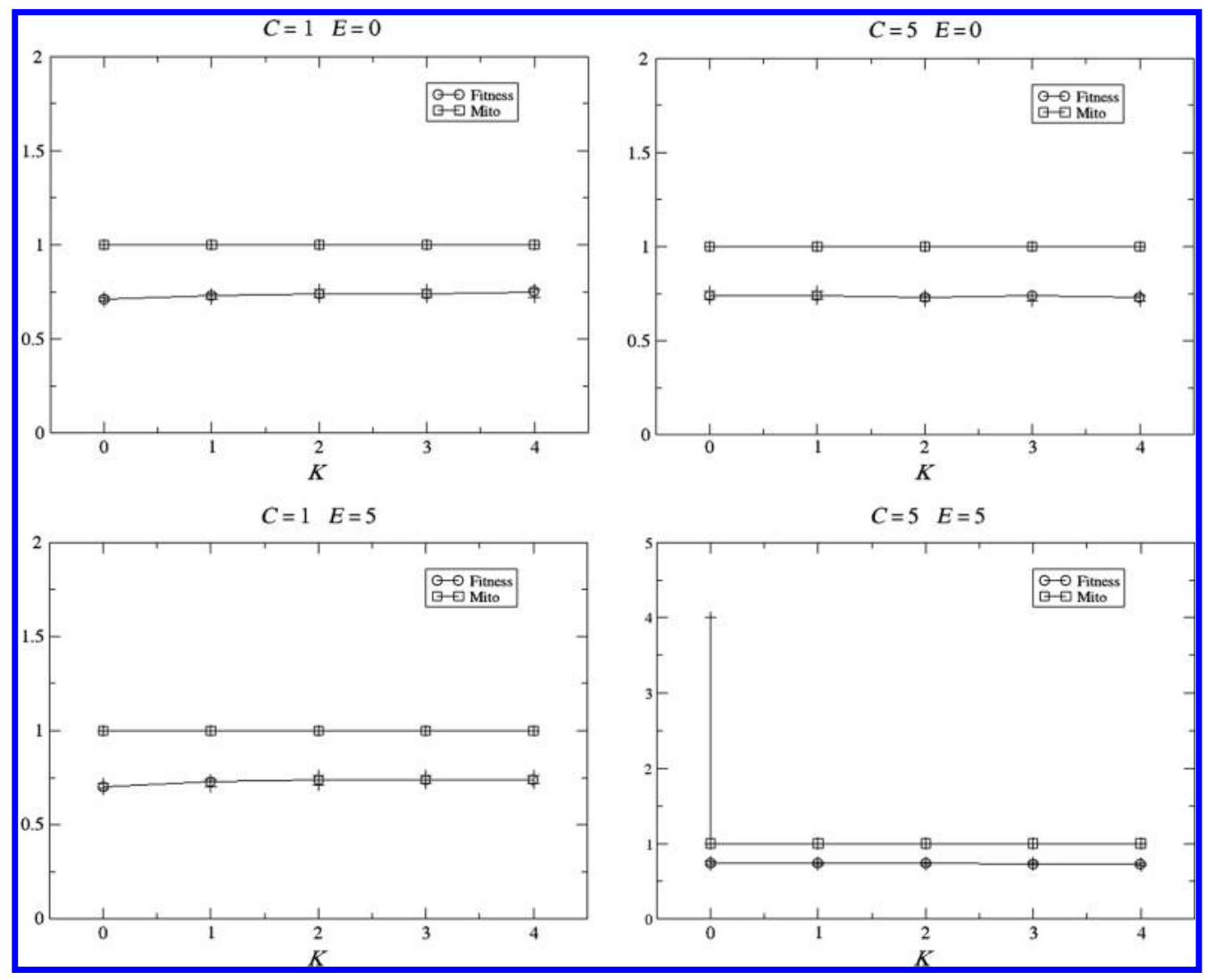

Figure 4. Example fitnesses and number of mitochondria across a variety of epistatic conditions within $(K)$ and between $(C)$ the nuclei/organelles, together with varying degrees of coupling to environment $(E)$. 

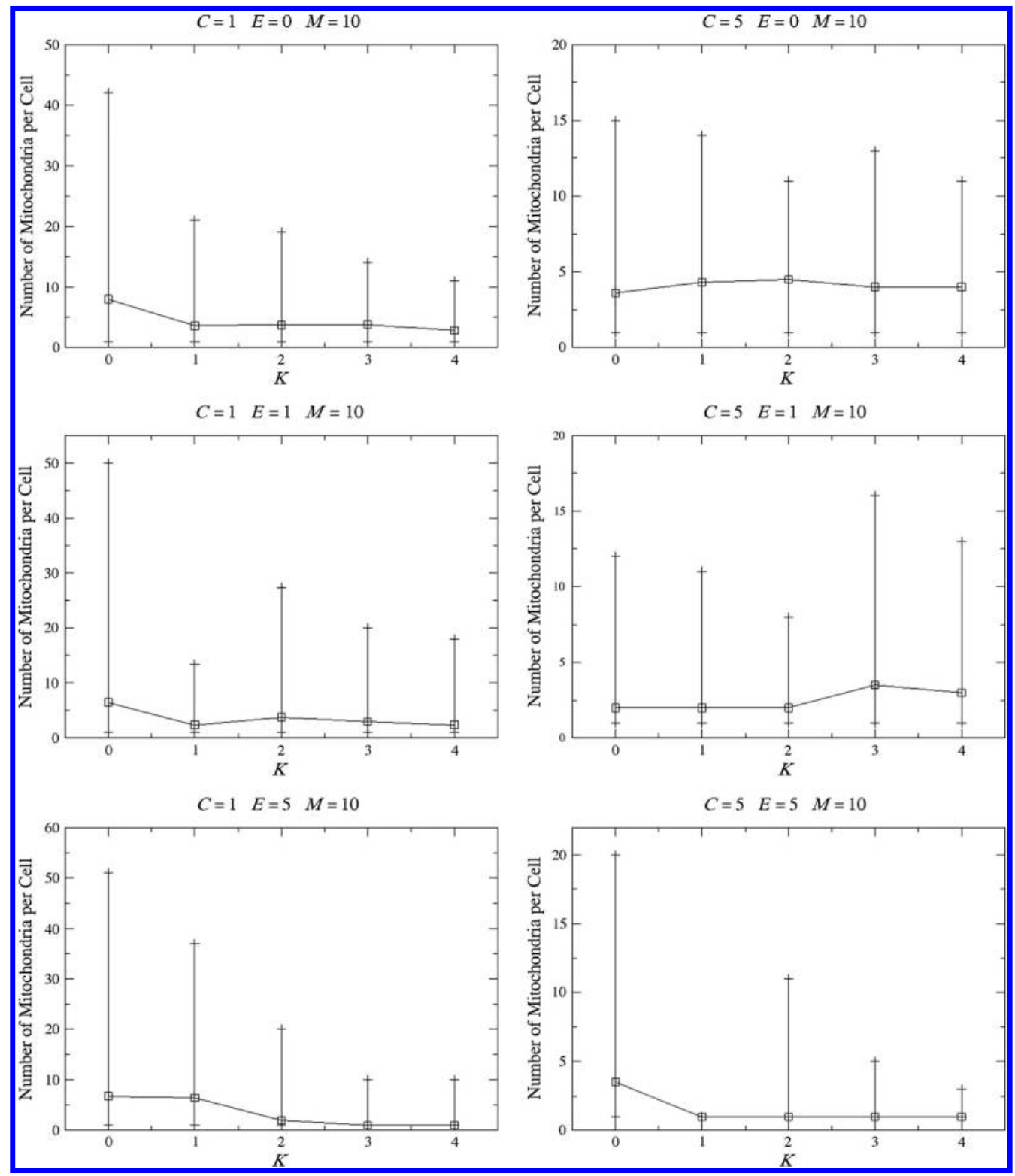

Figure 5. Example numbers of mitochondria across a variety of epistatic conditions within $(K)$ and between $(C)$ the nuclei/ organelles, with varying coupling to environment $(E)$ and a high rate of mitochondrial evolution $(M)$.

When $E=1$, the increase in $M$ does not significantly affect fitness, but more mitochondrial diversity is maintained for low $C(t$-test, $p<0.05)$. When $E=5$, increasing $M$ does not affect fitness but does increase the number of mitochondria for $K<2$ and $C=1$ ( $t$-test, $p<0.05$ ).

Similar results were found for $M=20$ (not shown).

\section{Conclusion}

Eukaryotic cells' ability to manipulate a population of mitochondria opens the potential for an evolutionary process occurring within the cytoplasm. Assuming a relatively simple self-monitoring/ 
selective mechanism exists, this letter suggests that the conditions under which this phenomenon emerges are widespread, sustained through a Baldwin-effect-like process, and correlated with those under which the prior symbiogenesis occurred. That is, the use of an intracellular evolutionary process appears to enable the beneficial maintenance of mitochondria upon multiple optima within the overall fitness landscape when that landscape is highly rugged.

It can also be noted that this result complements previous findings regarding the continued existence of mitochondrial DNA in spite of the transfer of over $90 \%$ of the original amount to the nucleus. It has been suggested that such horizontal gene transfer becomes less likely due to increasing drops in correlation in the fitness landscapes of the organelle before and after the move [3]. The Baldwin-like benefits of a heterogeneous population of mitochondria suggested here would also contribute to the cessation in transfers.

\section{References}

1. Baldwin, J. M. (1896). A new factor in evolution. American Naturalist, 30, 441-451.

2. Bull, L. (1999). On the Baldwin effect. Artificial Life, 5(2), 241-246.

3. Bull, L. (2010). Artificial symbiogenesis and differing reproduction rates. Artificial Life, 16(1), 65-72.

4. Bull, L., \& Fogarty, T. C. (1996). Artificial symbiogenesis. Artificial Life, 2(3), 269-292.

5. Burnet, F. M. (1976). A modification of Jerne's theory of antibody production using the concept of clonal selection. CA: A Cancer Journal for Clinicians, 26(2), 119-121.

6. Edelman, G. (1987). Neural Darwinism: The theory of neuronal group selection. Basic Books.

7. Issa, J.-P. (2011). Epigenetic variation and cellular Darwinism. Nature Genetics, 43(8), 724-726.

8. Kauffman, S. A. (1993). The origins of order: Self-organisation and selection in evolution. New York: Oxford University Press.

9. Kauffman, S. A., \& Johnsen, S. (1989). Co-evolution to the edge of chaos: Coupled fitness landscapes, poised states and co-evolutionary avalanches. In C. G. Langton, C. Taylor, J. D. Farmer, \& S. Rasmussen (Eds.), Artificial life II (pp. 325-370). Redwood City, CA: Addison-Wesley.

10. Keeler, K. (1985). Cost: benefit models of mutualism. In D. Boucher (Ed.), The biology of mutualism (pp. 100-127). London: Croom-Helm.

11. Kupiec, J.-J. (1996). A chance-selection model for cell differentiation. Cell Death and Differentiation, 3, 385-390.

12. Margulis, L. (1970). Origin of enkaryotic cells. Yale University Press.

13. Twig, G., Hyde, B., \& Shirihai, O. (2008). Mitochondrial fusion, fission and autophagy as a quality control axis: The bioenergetic view. Biochimica et Biophysica Acta-Bioenergetics, 1777(9), 1092-1097. 